In situ Electrocatalysis

\title{
In situ observation of surface species on iridium oxide nanoparticles during the oxygen evolution reaction**
}

\author{
Hernan G. Sanchez Casalongue, May Ling Ng, Sarp Kaya, Daniel Friebel, Hirohito Ogasawara, and \\ Anders Nilsson*
}

One of the crucial factors limiting efficient photo-induced water elctrocatalysis is the high overpotential required for the oxygen evolution reaction (OER). ${ }^{1,2}$ Of the many materials that can catalyze the OER, Iridium(IV) oxide has been shown to have high reactivity and excellent stability in both acidic and basic conditions ${ }^{3-6}$, but the complexity of the electrochemical process still presents many open questions about how this system operates.

First we need to understand the surface speciation of the catalyst under operating conditions. Competing suggestions exist about the chemical nature of Iridium(IV) oxide surfaces, ranging from $\mathrm{IrO}_{2}{ }^{6,7}$ to $\operatorname{Ir}(\mathrm{OH})_{4}{ }^{8}$, to mixtures of the two ${ }^{9}$. Previous theoretical ${ }^{10,11}$ and experimental ${ }^{9,11,12}$ efforts are also contradictory on the oxidation state of iridium during the OER process: One hypothesis 11,12, involving an $\operatorname{Ir}(\mathrm{V})$ intermediate, proposes the OER through a hydroperoxide mechanism, while the other 9,10 involves the deprotonation of two adjacent hydroxide groups on the same iridium site, resulting in a $\operatorname{Ir}(\mathrm{VI})$ intermediate. Secondly, it is essential to discern whether the catalytic process changes the chemical nature of the oxide only in the surface region or also in the bulk. Attempts to address these open questions have been made using many spectroscopic techniques ${ }^{13,14}$, yet the answers provided have not been fully satisfactory due to difficulties in performing direct, surface sensitive, in situ measurements.

Herein, we report on the use of synchrotron-radiation based Ambient Pressure Photoelectron Spectroscopy (APXPS) ${ }^{15,16}$,

[*] H. Sanchez Casalongue, Dr. S. Kaya, Dr. D. Friebel, Dr. A. Nilsson. Joint Center for Artificial Photosynthesis (JCAP) Energy Innovation Hub, LBNL, 1 Cyclotron Road, MS 976-JCAP, Berkeley, CA 94720. email: nilsson@slac.stanford.edu

H. Sanchez Casalongue, Dr. M. Ng, Dr. S. Kaya, Dr. D. Friebel, Dr. H. Ogasawara, Dr. A. Nilsson.

Sustainable Energy Through Catalysis (SUNCAT) Center for Interface Science and Catalysis, SLAC National Accelerator Laboratory, 2575 Sand Hill Rd, Menlo Park, CA 94025, USA.

Dr. H. Ogasawara, Dr. A. Nilsson.

Stanford Synchrotron Radiation Lightsource (SSRL), SLAC National Accelerator Laboratory, 2575 Sand Hill Rd, Menlo Park, CA 94025, USA.

[**] This material is based upon work performed by the Joint Center for Artificial Photosynthesis, a DOE Energy Innovation Hub, as follows: the experimental work was supported by the Joint Center for Artificial Photosynthesis award no. DE-SC0004993. H.O. gratefully acknowledges the support from Precursory Research for Embryonic Science and Technology (PRESTO), Japan Science and Technology Agency (JST). Portions of this research were carried out at the Stanford Synchrotron Radiation Lightsource (SSRL), a division of SLAC National Accelerator Laboratory and an Office of Science user facility operated by Stanford University for the U.S. Department of Energy.

Supporting information for this article is available on the WWW under http://dx.doi.org/10.1002/anie.201xxxxxx. together with a novel electrochemical cell, to provide operando measurements of the chemical speciation of the Iridium oxide surface during OER. We observe that, upon exposure to water, the catalyst surface shows clear signs of partial hydroxylation. We also show that, when the catalyst is evolving oxygen, there is a decrease in surface hydroxide species concurrent with the appearance of $\operatorname{Ir}(\mathrm{V})$, which indicates that the oxygen evolution reaction occurs on an $\operatorname{Ir}(\mathrm{V})$ site that would favor the $\mathrm{OOH}$ intermediate route. Furthermore, by tuning the photon energy of the X-rays, we are capable of probing different depths of the catalyst and determine that these reaction-induced changes are confined to the surface of the catalyst.
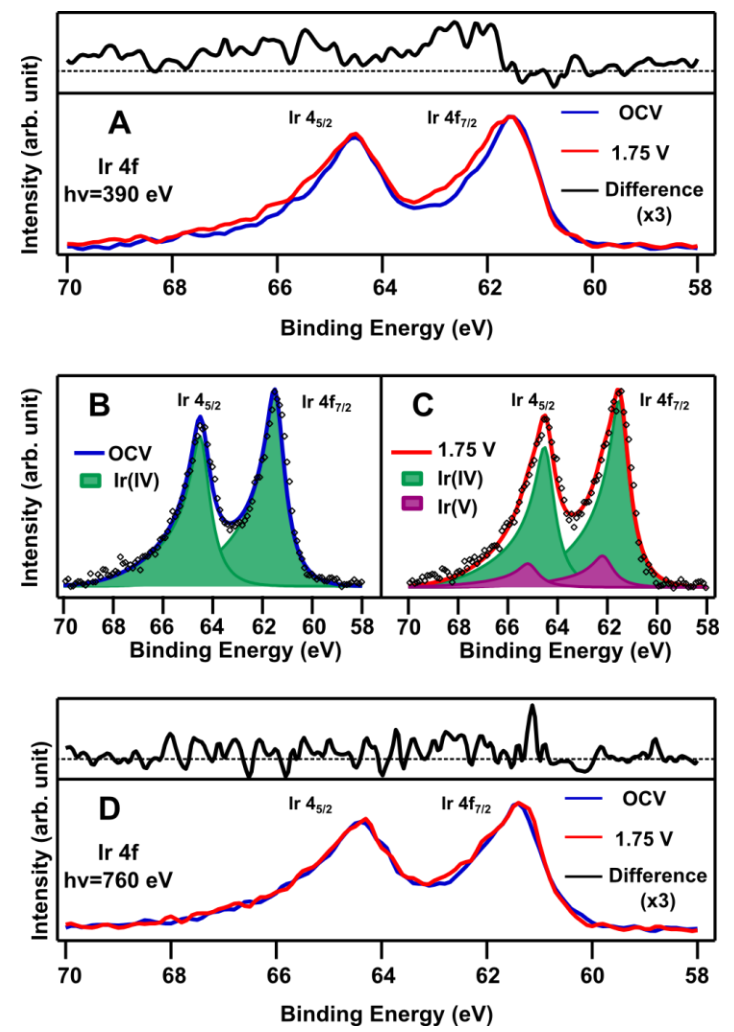

Figure 1. Ir $4 \mathrm{f}$ XPS spectra of iridium (IV) oxide nanoparticles under 10 Torr of water. (A) Ir $4 \mathrm{f}$ signal at open circuit voltage (Blue line) and during oxygen evolution reaction (Red line) collected with an incident photon energy of $390 \mathrm{eV}$. The black line above corresponds to the difference spectrum. (B) Curve fitted Ir 4f XPS spectrum under open circuit conditions, where the green components corresponds to $\operatorname{Ir}(\mathrm{IV})$. (C) Curve fitted Ir $4 \mathrm{f}$ XPS spectrum under oxygen evolution conditions. The green and purple components correspond to $\operatorname{Ir}(\mathrm{IV})$ and $\operatorname{Ir}(\mathrm{V})$, respectively (D) Ir $4 \mathrm{f}$ signal measured at open circuit voltage (Blue line) and during oxygen evolution (Red line) conditions collected with an incident photon energy of $760 \mathrm{eV}$. The black line above corresponds to the difference spectrum. 
Building on previous operando electrochemical APXPS studies ${ }^{17}$, the experimental setup consisted of a polymer electrolyte membrane (PEM) electrochemical cell designed to work compatibly with APXPS (SI 1). The working electrode side of the membrane was coated with Iridium(IV) oxide nanoparticles and the counter electrode with platinum nanoparticles (see Experimental Section and SI 2). The assembled cell was introduced to the APXPS system and hydrated with a pressure of 10 Torr of water, at which point electrochemical characteristics were measured, whereby the onset of OER at the working electrode was observed at an applied voltage of around 1.6 V (SI 3).

Fig 1A shows the Ir 4f spectra of the electrode surface probed with an incident photon energy of $390 \mathrm{eV}$ under a water vapor pressure of 10 Torr at open circuit voltage (OCV, blue) and at an OER operating voltage of $1.75 \mathrm{~V}$ (red). As voltage is applied, we observe an increase in intensity at the higher binding energy (BE) regions of both the Ir $4 \mathrm{f}_{5 / 2}$ and $\operatorname{Ir} 4 \mathrm{f}_{7 / 2}$ components, as shown in the difference spectrum (black). This difference is accounted by two distinctive features at BEs of 62.2 and $65.2 \mathrm{eV}$, which we attribute to the $\operatorname{Ir} 4 \mathrm{f}_{5 / 2}$ and $\operatorname{Ir} 4 \mathrm{f}_{7 / 2}$ components of iridium atoms that have undergone chemical changes under reaction conditions. This change is reversible with applied voltage, only appearing under oxygen evolution conditions. No changes were observed in the Ir $4 \mathrm{f}$ spectra upon humidification. Figure 1A is curve fitted in Figures 1B and 1C (See Experimental Section). When no current is applied, the open circuit voltage remains under $100 \mathrm{mV}$ and the iridium spectra can be fitted with a single set of components at 61.5 and $64.5 \mathrm{eV}$ (green), in agreement with reported BE values for $\operatorname{Ir}(\mathrm{IV}){ }^{7}$ This single set of components also rules out contributions from metallic iridium, $\operatorname{Ir}(0)$, with a reported $\mathrm{BE}$ of $60.9 \mathrm{eV} .{ }^{9}$ Using the fitting parameters obtained from OCV conditions, it was not possible to recreate the line shape of the spectra under OER conditions, shown in Figure 1C, unless a second set of higher BE components was added into the fit at 62.2 and $65.2 \mathrm{eV}$ (purple). Therefore both the fit and the difference spectrum support the hypothesis of two distinct Iridium oxidation states being present on the catalyst under reaction conditions.

A chemical shift in the XPS BE towards higher values corresponds to an increase in the oxidation state, as observed in previous works ${ }^{8,9}$ and well known for metals and oxides. ${ }^{18}$ Considering that platinum $(\mathrm{Pt})$ and iridium are neighboring elements in the periodic table, we can compare the current results with similar spectral changes in platinum and platinum oxides ${ }^{19}$ : For a $\operatorname{Pt}(111)$ surface, the $\mathrm{BE}$ shift between $\mathrm{Pt}(0)$ and $\mathrm{Pt}(\mathrm{II})$ is $0.7 \mathrm{eV}$, while the shift between $\mathrm{Pt}(\mathrm{II})$ and $\mathrm{Pt}(\mathrm{IV})$ is $2.5 \mathrm{eV}$, suggesting larger BE shifts for higher oxidation state transitions. This trend is corroborated by molybdenum and tungsten oxides, with BE shifts of 4 and $4.3 \mathrm{eV}$ between the (IV) and (VI) oxidation states ${ }^{20}$, and by iridium oxide itself, with a reported BE shift of $1.4 \mathrm{eV}$ between $\operatorname{Ir}(\mathrm{IV})$ and $\operatorname{Ir}(\mathrm{VI}){ }^{8}$ Based on the difference spectra from Figure 1A and the fit from Figure 1C, the new iridium chemical state has a BE $0.7 \mathrm{eV}$ higher than the $\operatorname{Ir}(\mathrm{IV})$ component, which indicates an oxidation state higher than (IV) but lower than (VI). This suggests that the new iridium feature corresponds to an apparent oxidation number of only one unit higher, i.e. $\operatorname{Ir}(\mathrm{V})$, under OER conditions. The relative ratios of the components of Figure 1C indicate that $14.6 \%$ of the $\operatorname{Ir}(\mathrm{IV})$ within the probing depth $(\sim 0.7 \mathrm{~nm})$ is transformed into $\operatorname{Ir}(\mathrm{V})$ under reaction conditions, which suggests that only a fraction of the surface iridium atoms have been transformed during OER.

In addition to chemical speciation, surface and bulk contributions can be differentiated by changing the depth sensitivity.
Figure 1D shows the Ir $4 \mathrm{f}$ spectra of the electrode surface with (red) and without (blue) applied voltage at a photon energy of $760 \mathrm{eV}$. The increase in kinetic energy of the emitted photoelectrons from $325 \mathrm{eV}$ to $700 \mathrm{eV}$ corresponds to a doubling of the photoelectron inelastic mean free path, from approximately $0.7 \mathrm{~nm}$ to $1.1 \mathrm{~nm}$ (Calculated, see Experimental Section). The difference spectrum shown in Figure 1D (black) still indicates the growth of a higher binding energy feature, but the change is more subtle. Therefore, we propose that the growth of Iridium(V) is limited to the top surface layer of the catalyst, indicating that the transformation of $\operatorname{Ir}(\mathrm{IV})$ into $\operatorname{Ir}(\mathrm{V})$ plays a much larger role than the $14.6 \%$ in Figure 1C would indicate at first: If we were to restrict all the iridium changes to the top layer of the Iridium, the $\operatorname{Ir}(\mathrm{V})$ could represent as much as one third of the surface iridium atoms.
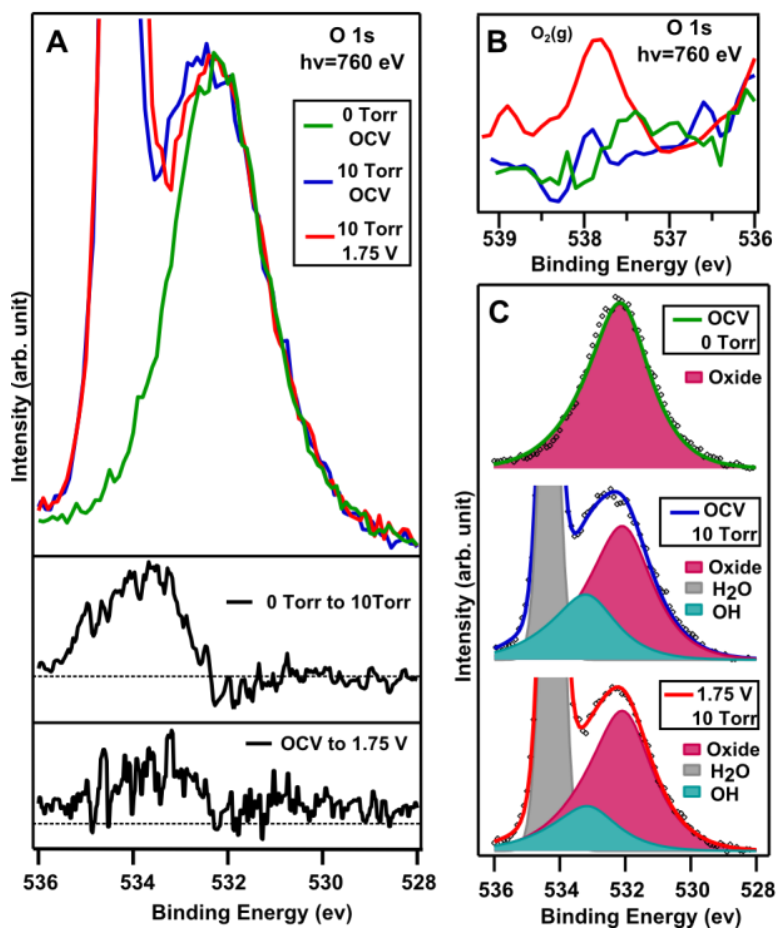

Figure $201 s$ spectra of iridium oxide nanoparticles collected with an incident photon energy of $760 \mathrm{eV}$. (A) $O$ 1s signal measured under vacuum (green) and under 10 Torr water pressure at open circuit voltage (blue) and during oxygen evolution reaction (red). The black lines below correspond to the difference spectra. (B) Higher binding energy region of the $O$ 1s XPS spectra shown in (A). The new component corresponds to gas phase oxygen being evolved from the catalyst. (C) Curve fitted O 1s XPS spectra under vacuum (green), open circuit voltage (blue) and oxygen evolution conditions (red). The grey, teal and magenta components correspond to water, hydroxide and oxide components, respectively.

Figure 2A shows the $\mathrm{O} 1 \mathrm{~s}$ spectra of the iridium oxide nanoparticle surface, probed with a photon energy of $760 \mathrm{eV}$ for a kinetic energy of $225 \mathrm{eV}$, under vacuum (green), under 10 Torr of water at OCV (blue), and under both 10 Torr of water and $1.75 \mathrm{~V}$ applied voltage (red). The two difference spectra (black) reveal that there are spectral changes as the surface is taken from vacuum to OCV and to OER conditions. There is also another spectral feature at much higher binding energy, shown at $537.8 \mathrm{eV}$ in Figure 2B, which appears only during OER conditions. This peak corresponds to gas phase oxygen $17,21,22$, confirming the electrochemical formation of oxygen during the XPS measurements. 
The spectra shown in Figure 2A are curve fitted in Figure 2C. In the OCV and OER O 1s spectra, the intensity of the water gas phase and liquid peak $(\mathrm{BE}=534.3 \mathrm{eV})$ has been truncated to better highlight the relative changes in the surface species. We can differentiate three separate oxygen-containing species through their distinctive binding energies: The first peak, at $532.1 \mathrm{eV}$ (magenta) and the only component of the pristine sample, corresponds to the $\mathrm{O}_{2}$ - anions of surface oxides with their oxygen atoms being the most screened due to the bonding with iridium. Second, we observe hydroxide groups on the surface at a BE of $533.2 \mathrm{eV}$ (teal), consistent with the work in ref. 8 and 9. The last species at $534.3 \mathrm{eV}$ (grey) corresponds to water, with signals from adsorbed water and bulk electrolyte overlapping with the gas phase component, which appears at a lower BE than other works ${ }^{23-25}$ due to the applied voltage. ${ }^{17} \mathrm{OOH}$ was not included in the fit since its two characteristic features, 1 and $2.5 \mathrm{eV}$ higher $\mathrm{BE}$ than $\mathrm{OH}{ }^{17,26}$, would overlap with and be masked by the much larger water component. Although our observed BE values are slightly higher than observed previously ${ }^{8}$ - likely due to challenges with the absolute $\mathrm{BE}$ calibration (see Experimental Section) -, the energy separation between species is consistent with previous works ${ }^{23,24,27}$ with the strongly bonded oxides having the lowest $\mathrm{BE}$ and the loosely associated water molecules the highest BE. We observe that, as water is introduced and the iridium nanoparticles reach open circuit conditions, part of their surface is covered by adsorbed hydroxide, resulting in a 1:2 hydroxide to oxide ratio (by area). Since the Ir $4 \mathrm{f}$ signal did not change upon humidification, this means that oxygen atoms are partially transformed to surface hydroxide without changing the oxidation state of Iridium. When the system is taken to OER conditions, the ratio of the areas decreases to $1: 3$, corresponding to a reduction in the amount of adsorbed hydroxide present on the surface. These changes are consistent with previous theoretical works ${ }^{11}$, which postulate that surface hydroxide will be stable on iridium oxide at potentials below $1.5 \mathrm{~V}$ vs. RHE, above which the O-containing species become more stable and the surface will be able to undergo the deprotonation step that triggers the oxygen evolution reaction.

Therefore, our results provide three key findings that will add to the fundamental understanding of the oxygen evolution reaction on iridium oxide nanoparticles: We show that iridium oxide nanoparticles in contact with water exhibit a mixed surface composition, with both oxide and hydroxide species coexisting at the same time. We can confirm that the oxygen evolution reaction takes place through a deprotonation mechanism ${ }^{9}$ in which hydroxide is converted into oxide on the catalyst surface. As this conversion takes place, we identified the change in the oxidation number of $\operatorname{Ir}$ from $\operatorname{Ir}(\mathrm{IV})$ to $\operatorname{Ir}(\mathrm{V})$. Therefore, we are able to favor a mechanism in which the OER takes place through an $\mathrm{OOH}$ intermediate, where the $\operatorname{Ir}(\mathrm{V})$ becomes the energy sink in the reaction since a higher free energy change is required to form the $\mathrm{OOH}$, in agreement with the efforts by Rossmeisl et al. ${ }^{11}$ Finally, we are able to show that the changes in iridium only take place at the top surface layers, with no chemical changes taking place at the bulk of the catalyst.

In conclusion, through to the combination of electrochemistry and APXPS, we have probe the surface of iridium oxide nanoparticles during oxygen evolution reaction conditions. We have show that, in the presence of electrolyte, the surface is covered by a mixture of oxide and adsorbed hydroxide species. We also have found experimental evidence that strongly suggests that the oxygen evolution takes place through the creation of iridium oxide intermediates with an apparent oxidation state of $\operatorname{Ir}(\mathrm{V})$, but only on the outer layers of the catalyst surface. The presence of Iridium(V) as opposed to Iridium(VI) indicates that the water splitting process occurs on a single iridium site through an $\mathrm{OOH}$ intermediate, clarifying the OER mechanism on an iridium catalyst. These results show the versatility of APXPS electrochemistry, and we believe this new technique will open the door to unprecedented fundamental understanding of reaction mechanisms for all electrochemical processes relevant for solar to fuel conversion.

\section{Experimental Section}

For the XPS measurements, the electrochemical cell was placed in the gas cell of the APXPS system on beamline 13-2 at the Stanford Synchrotron Radiation Lightsource (SSRL). Depending on the desired measurement, the incident photon energy was set to either $390 \mathrm{eV}$ or $760 \mathrm{eV}$ : These specific energies were chosen to maximize the surface sensitivity in water ambient.

The working electrode was prepared by coating the front side of the membrane with $0.08 \mathrm{mg} / \mathrm{cm} 2$ Iridium (IV) Oxide nanoparticles $(50-80 \mathrm{~nm}$ in diameter, Nanograde Inc.) through a one-step drop-casting method ${ }^{28}$ (The electrode characterization is shown in SI 2). The counter electrode on the back side of the membrane consisted of platinum $(\mathrm{Pt})$ nanoparticles supported on a Nafion $® /$ carbon-black mixture. The assembled cell was introduced into the APXPS system, where saturated water vapor was introduced to the counter electrode through small tubes, while the working electrode side exposed to the gas cell was humidified via variable leak valves. The water vapor pressure was set to 10 Torr as to achieve a relative humidity of approximately $50 \%$ at room temperature and provide electrolyte for electrolysis. Once the system was humidified, its electrochemical characteristics were assessed through twoelectrode cyclic voltammetry (CV) using a PineWave Potentiostat, as shown in SI 3. All XPS and electrochemical measurements were performed at room temperature.

For the Ir $4 f$ spectra, the XPS BE is referenced to the Fermi edge under the corresponding experimental conditions and the signal intensity is normalized to the $\operatorname{Ir} 4 f 7 / 2$ intensity. Due to a small amount of surface charging during the acquisition of the $O$ 1s spectra (seen in the lower $B E$ of the gas phase water), the O1s BE is not referenced to the Fermi level but to the lower BE onset of the signal. The O1s intensity is normalized to the signal background. Figure SI 2C shows an overview XPS spectrum of the anode surface.

Spectral deconvolutions and background subtractions (Shirley Backgrounds) were performed using lgor-Pro software. The OCV Ir $4 f$ spectrum was deconvoluted using Gaussian-broadened Doniach-Šunjić functions (FWHM = 1, 85\% Gaussian- Lorentzian, 0.9 asymmetry). Ir spectrum recorded in OER conditions was deconvoluted using the parameters from the open circuit conditions, with a second higher binding energy component being added to produce a good fit. $\mathrm{O}$ 1s spectra under both open circuit and oxygen evolution conditions were deconvoluted using Voigt functions (FWHM $=2.2,75 \%$ Gaussian- Lorentzian, 0.57asymmetry).The Inelastic Mean Free Path was calculated using the NIST Electron Inelastic-Mean-Free-Path Database: Version 1.2 , using the predictive G-1 equation of Gries et al.

Received: ((will be filled in by the editorial staff))

Published online on ((will be filled in by the editorial staff))

\section{Keywords: Catalysis $•$ Electrochemistry $\cdot$ APXPS $\cdot$ OER $・$ Iridium Oxide}

[1] J. McKone, N. Lewis, B. Gray. Chem. Mater. 2013, DOI: 10.1021. 


\section{Angewandte}

\section{Communications}

[2] B. Pinaud, T. Jaramillo, et al. Energy Environ. Sci. 2013, 6, 1983 2002

[3] C. McCrory, et al. J. Am. Chem. Soc., 2013, 135, 16977-16987.

[4] Th. Pauporté, F. Andolfatto, R. Durand. Electrochimica Acta 1999, 45, 431-439.

[5] P. Steegstra, E. Ahlberg. Journal of Electroanalytical Chemistry 2012, $685,1-7$.

[6] M. Yagi, E. Tomita, T. Kuwabara. Journal of Electroanalytical Chemistry 2005, 579, 83-88.

[7] M. Bozack. Surf. Sci. Spectra 1993, 2, 123.

[8] J. Augustynski, M. Koudelka, J. Sanchez. Journal of Electroanalytical Chemistry and Interfacial Electrochemistry 1984, 160, 233-248.

[9] R. Kötz, H. Neff, S. Stucki. J. Electrochem. Soc: Electrochemical science and technology 1984, 131, 72-77.

[10] M. Lyons, L. Burke. Phys. Chem. Chem. Phys. 2011, 13, 5314-5335.

[11] J. Rossmeisl, J. Nørskov, et al. Journal of Electroanalytical Chemistry 2007, 607, 83-89.

[12] P. Steegstra, E. Ahlberg. Electrochimica Acta 2012, 76, 26-33.

[13] R. Hillman, M. Skopek, S. Gurman. Phys. Chem. Chem. Phys 2011 $13,5252-5263$.

[14] Y. Mo, A. Scherson, et al. J. Phys. Chem. B 2002, 106, 3681-3686.

[15] S. Kaya, A. Nilsson, et al. Catal. Today 2013, 205, 101-105.

[16] M. Salmeron, R. Schlögl. Surf. Sci. Rep. 2008, 63, 169-199.

[17] H. Sanchez Casalongue, A. Nilsson, et al. Nature Comm. 2013, DOI:10.1038/ncomms3817
[18] S. Hufner, in Photoelectron spectroscopy: Principles and applications. 3rd edition, Springer-Verlag 2003.

[19] D. Miller, A. Nilsson, et al. Phys. Rev. Lett. 2011, 107, 195502.

[20] D. Sarma, C., Rao. Journal of Electron Spectroscopy and Related Phenomena 1980, 20, 25-45.

[21] H. Bluhm, R., Schlögl, et al. J. Phys. Chem. B 2004, 108, 1434014347.

[22] S. Blomberg , E., Lundgren, et al. Phys. Rev. Lett. 2013, 110 117601.

[23] J. Newberg, H., Bluhm, et al. Surface Science 2011, 605, 89-94.

[24] S. Yamamoto, A. Nilsson, et al. J. Phys. Chem. C 2010, 114, 22562266

[25] S. Sunde, I. Lervik, M. Tsypkin, L. Owe. Electrochimica Acta 2010, $55,7751-7760$.

[26] J. MacNaughton, A. Nilsson, et al. Phys. Chem. Chem. Phys. 2010, $12,5712-5716$.

[27] T. Kendelewicz, G. Brown, et al. J. Phys. Chem. C 2013, 117, 27192733

[28] T. Reier, M. Oezaslan, P. Strasser. ACS Catal. 2012, 2, 1765-1772. 


\section{Angewandte \\ Communications}

Entry for the Table of Contents

\section{In situ Electrocatalysis}

Hernan G. Sanchez Casalongue, May Ling Ng, Sarp Kaya, Daniel Friebel, Hirohito Ogasawara, and Anders Nilsson*

\section{Page - Page}

The surface species of an iridium oxide electrocatalyst under oxygen evolution reaction (OER) conditions were probed in-situ through Ambient Pressure X-ray Photoelectron Spectroscopy. We demonstrate that, under OER conditions, iridium undergoes a change in oxidation state from $\operatorname{Ir}(\mathrm{IV})$ to $\operatorname{Ir}(\mathrm{V})$ that takes place predominantly at the surface of the catalyst, suggesting that the oxygen evolution reaction on iridium oxide occurs through an $\mathrm{OOH}$ mediated deprotonation mechanism.
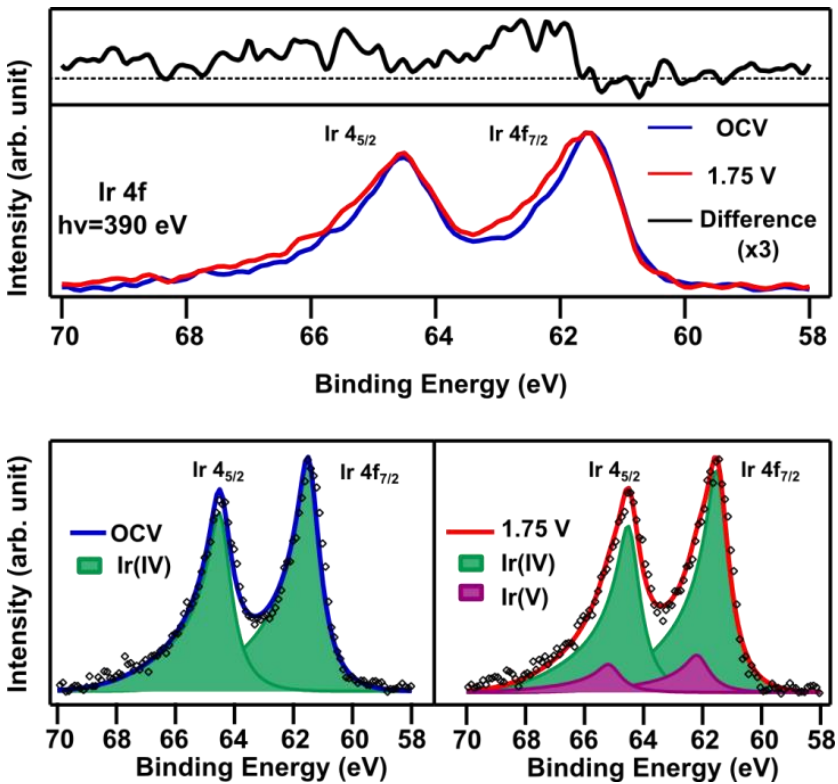